\title{
$\square$ CARTA AL EDITOR
}

\section{Un caso de replicación mediante el factor Bayes en las ciencias de la salud}

\author{
A case of replication using the Bayes factor \\ in the health sciences
}

\section{Cristian Antony Ramos-Vera ${ }^{1}$}

${ }^{1}$ Universidad César Vallejo, Facultad de ciencias de la salud, Área de investigación. Lima, Perú.

\section{Señor Editor}

En el número 1 del volumen 7 de la Revista virtual de la Sociedad Paraguaya de Medicina Interna se publicó un importante estudio que reporta la existencia de una correlación positiva y estadísticamente significativa entre el índice de masa corporal (IMC) y la presión arterial diastólica (PAD), en 170 pacientes con diagnóstico de cáncer de mama (1). Estos datos fueron analizados mediante la significación estadística de la hipótesis nula (NHST, siglas en inglés) "p<0,05", utilizando el coeficiente de correlación de Spearman, que infiere el rechazo de la hipótesis nula (no correlación) y brinda mayor confianza de verosimilitud al investigador a asumir la hipótesis alterna (correlación).

Se recomienda la replicación de las investigaciones en ciencias de la salud basadas en el enfoque NHST para generar una evidencia concluyente con mayor credibilidad (2). Esto es posible mediante la inferencia bayesiana, pues permite reanalizar el hallazgo significativo reportado en este artículo, donde el método del factor de Bayes es referido como la probabilidad de los datos bajo una hipótesis en relación con la otra (hipótesis nula vs hipótesis alterna) ${ }^{(3,4)}$. Es decir, que el factor Bayes estima la cuantificación del grado o evidencia en que los datos apoyan tanto la hipótesis nula como la hipótesis alterna para su contraste más allá de la interpretación dicotómica del rechazo o aceptación de la hipótesis nula (NHST) (3-5). La interpretación está basada en el esquema de clasificación de valores de Jefreys ${ }^{(3-5)}$ : "débil", moderado", "fuerte" "muy fuerte" y "extrema" (tabla 1).

Tabla 1. Valores de interpretación cuantificable del factor Bayes

\begin{tabular}{lll}
\hline$>100$ & Extrema & Hipótesis alternativa \\
$30-100$ & Muy fuerte & Hipótesis alternativa \\
$10-30$ & Fuerte & Hipótesis alternativa \\
$3,1-10$ & Moderado & Hipótesis alternativa \\
$1,1-3$ & Débil & Hipótesis alternativa \\
1 & 0 & No evidencia \\
$0,3-0,9$ & Débil & Hipótesis nula \\
$0,29-0,1$ & Moderado & Hipótesis nula \\
$0,09-0,03$ & Fuerte & Hipótesis nula \\
$0,03-0,01$ & Muy fuerte & Hipótesis nula \\
$<0,01$ & Extrema & Hipótesis nula \\
\hline
\end{tabular}

Nota: Creación propia según la escala de clasificación de Jeffreys ${ }^{(4)}$

\section{Autor correspondiente:}

Dr. Cristian Antony Ramos-Vera

Miembro de la Sociedad Peruana de Psicometría

Correo electrónico: cristony_777@hotmail.com

Artículo recibido: 28 noviembre 2020 Artículo aceptado: 4 diciembre 2020 
Se tuvo como fin de la presente carta reportar un ejemplo de reanálisis bayesiano para comprobar la hipótesis alterna (correlación) (2). Se consideró los datos del tamaño de muestra y el coeficiente de correlación Spearman de IMC-PAD $(0,15)$ reportado por Ayala et al. (1). El factor Bayes considera dos interpretaciones: $\mathrm{FB}_{10}$ (a favor de la hipótesis alternativa de significancia) y $\mathrm{BF}_{01}$ (a favor de la hipótesis nula), con un intervalo de credibilidad del $95 \%{ }^{(5-8)}$.

Los resultados obtenidos del factor Bayes evidenciaron que $\mathrm{BF}_{10}=6,614$ y $\mathrm{BF}_{01}=0,151 \mathrm{e}$ IC95\% $[0,048-0,247]$, lo que respaldó los resultados con respecto a la relación estadística entre IMC-PAD reportada por Ayala et al. (1), mientras la estimación de probabilidad bayesiana de la hipótesis nula $\left(\mathrm{BF}_{01}\right)$ fue proporcionalmente menor en contraste a la hipótesis alterna (6 veces mayor).

Así también, se reporta el parámetro del factor Bayes máximo ( $\operatorname{maxBF}_{10}=14,23$ ) para determinar la estabilidad de los resultados, cuya estimación de mayor magnitud de respaldo al hallazgo significativo de correlación fortalece la confiabilidad de la inferencia bayesiana ${ }^{(3,5)}$.

El factor Bayes es de gran utilidad en otros análisis y reanálisis estadísticos que se basan en el enfoque NHST " $p<0,05$ " (prueba $t$, regresión lineal, regresión logística, ANOVA), siendo de gran relevancia la difusión del uso e interpretación del factor Bayes en las ciencias de la salud (79). Adicionalmente permite reforzar las investigaciones cuantitativas sistemáticas que usen dichas pruebas estadísticas para una mayor credibilidad en las conclusiones de estudios meta-analíticos. Por lo tanto, se considera esta carta como un aporte metodológico inclusivo para futuros artículos en la presente revista.

\section{Conflictos de interés}

El autor no declara conflictos de interés

\section{REFERENCIAS BIBLIOGRÁFICAS}

1. Ayala León SJ, Cabral F, Escalada G, Cabral L, Ayala León M, Gauna C. Factores de riesgo cardiovascular en pacientes con cáncer de mama ¿Existe correlación con las referencias internacionales? Rev. Virtual Soc. Parag. Med. Int. 2020; 7(1):66-76.

2. Ly A, Raj A, Etzn A, Marsman M, Gronau QF, Wagenmakers E-J. Bayesian reanalyses from summary statistics: a guide for academic consumers. Adv Meth Pract Psychol Sci /Internet/. 2018 /cited 2020 Feb 10/; 1(3):367-74. Available from: https://journals.sagepub.com/doi/ full/10.1177/2515245918779348.

3. Goss-Sampson MA. Bayesian inference in JASP: A guide for students /Internet/. Amsterdam: Johnny Van Doorn \& EJ Wagenmakers, JASP; 2020. /cited 2020 Feb 15/. Available from: http:// static.jasp-stats.org/ Manuals /Bayesian_Guide_v0_12_2_1.pdf. http://dx.doi.org/10.17605/ OSF.IO/CKNXM.

4. Jeffreys H. Theory of probability. Oxford: Oxford University Press; 1961.

5. Marsman M, Wagenmakers EJ. Bayesian benefits with JASP. Eur. J. Dev. Psychol /Internet/. 2017 /cited 2020 Marzo 3/; 14(5):545-55. Available from:https://www.tandfonline.com/doi/full/ $10.1080 / 17405629.2016 .1259614$.

6. Nuzzo RL. An introduction to Bayesian data analysis for correlations. PM\&R. 2017;9(12):127882.https://doi.org/10.1016/j.pmrj.2017.11.003 .

7. Quintana DS, Williams DR. Bayesian alternatives for common null-hypothesis significance tests in psychiatry: a non-technical guide using JASP. BMC Psychiatry /Internet/. 2018 /cited 2020 Abr 5/; 18(1):178. Available from: https://www.ncbi.nlm.nih.gov/pmc/articles/PMC5991426/.

8. Kelter R. Bayesian alternatives to null hypothesis significance testing in biomedical research: a non-technical introduction to Bayesian inference with JASP. BMC Med Res Methodol. 2020; 20(142):1-12. https://doi.org/10.1186/s12874-020-00980-6

9. Ramos-Vera C. Métodos estadísticos modernos para evaluar diferencias en el contexto pandémico: el caso del género en el miedo a la COVID-19. Rev Cubana Inv Bioméd. En prensa 